Special Section: The Root

Zone: Soil Physics and Beyond

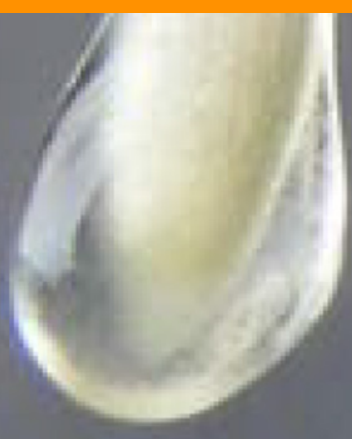

Core Ideas

- Mucilage exudation by roots modifies the hydraulic properties of the rhizosphere.

- Rhizoligands are surfactants that

rewet the rhizosphere and reduce

mucilage swelling.

- A reduction in mucilage swell-

ing reduces rhizosphere hydraulic

conductivity.

- By managing rhizosphere hydraulic

properties, we can improve plants'

adaptation to drought.

M.A. Ahmed, M. Zarebanadkouki, K. Ahmadi, and A. Carminati, Division of Soil Hydrology, Georg-August Univ. of Göttingen, Göttingen, Germany; M.A. Ahmed, Dep. of Agricultural Engineering, Univ. of Khartoum, Khartoum, Sudan; E. Kroener, Group of Geophysics, Univ. of Koblenz-Landau, Landau, Germany; S. Kostka, Aquatrols Corporation of America, Paulsboro, NJ 08066; A. Kaestner, Lab. for Neutron Scattering and Imaging, Paul Scherrer Institute, Villigen, Switzerland. *Corresponding author (mahmed@gwdg.de).

Received 29 Sept. 2016

Accepted 14 Nov. 2016.

Supplemental material online.

Citation: Ahmed, M.A., M. Zarebanadkouki, K. Ahmadi, E. Kroener, S. Kostka, A. Kaestner, and A. Carminati. 2018. Engineering rhizosphere hydraulics: Pathways to improve plant adaptation to drought. Vadose Zone J. 17:160090. doi:10.2136/vzj2016.09.0090

(c) Soil Science Society of America.

This is an open access article distributed

under the CC BY-NC-ND license

(http://creativecommons.org/licenses/

by-nc-nd/4.0/).

\section{Engineering Rhizosphere Hydraulics: Pathways to Improve Plant Adaptation to Drought}

\author{
Mutez A. Ahmed,* Mohsen Zarebanadkouki, Katayoun \\ Ahmadi, Eva Kroener, Stanley Kostka, Anders Kaestner, \\ and Andrea Carminati
}

Recent studies have drawn attention to the role of mucilage in shaping rhizosphere hydraulic properties and regulating root water uptake. During drying, mucilage keeps the rhizosphere wet and conductive, but on drying it turns hydrophobic, limiting root water uptake. In this study, we introduce the concept of rhizoligands, defined as additives that (i) rewet the rhizosphere and (ii) reduce mucilage swelling, thereby reducing the rhizosphere conductivity. We tested whether selected surfactants behaved as rhizoligands. We used neutron radiography to monitor water redistribution in the rhizosphere of lupine (Lupinus albus L. CV. Feodora) and maize (Zea mays L.) irrigated with water and rhizoligands. In a parallel experiment, we tested the effect of rhizoligands on the transpiration rate of lupine and maize subjected to repeated drying and wetting cycles. We also measured the effect of rhizoligands on the maximum swelling of mucilage and the saturated hydraulic conductivity of soil mixed with various mucilage concentrations. Rhizoligand treatment quickly and uniformly rewetted the rhizosphere of maize and lupine. Interestingly, rhizoligands also reduced transpiration during drying-wetting cycles. Our hypothesis is that the reduction in transpiration was triggered by the interaction between rhizoligand and mucilage exuded by roots. This hypothesis is supported by the fact that rhizoligand reduced the maximum swelling of mucilage, increased its viscosity, and decreased the hydraulic conductivity of soil-mucilage mixtures. The reduced conductivity of the rhizosphere induced a moderate stress to the plants, reducing transpiration. Rhizoligands increase the rhizosphere wetting kinetics and decrease the maximum swelling of mucilage. As a consequence, root rehydration following irrigation is faster, a larger volume of water is available to the plant, and this water is used more slowly. This slower water consumption would allow the plant to stay turgid during a prolonged drying period. We propose that by managing the hydraulic properties of the rhizosphere, we can improve plants' adaptation to drought.

A major challenge to agriculture in the 21st century is to make the most efficient possible use of water resources. Modern agricultural production has been based on the wide availability of input resources such as fertilizer and water (Lynch and Brown, 2012), but it is now generally accepted that a shortage of water is one of the greatest constraints to meeting the world's inevitably increasing demand for food as the world's population continues to grow. Increasing plant drought tolerance and improving the capacity of agricultural crops to extract water from the soil are therefore essential research targets.

Many propose the use of genetic modification to improve water efficiency in crop production. However, genetically modified crops may have unknown evolutionary consequences for ecosystems. Furthermore, such genetic manipulations are expensive, resulting in seed that often is not affordable for farmers in most developing countries. Alternative strategies for enhancing crop production are therefore urgently being sought. 
One approach recently put forward to improve crop productivity is the use of plant-soil feedbacks, which can improve the capacity of plant roots to extract water from the soil (Sposito, 2013). Several root traits are involved in extracting water from soil, including root architecture (Wasson et al., 2012), root depth (Tron et al., 2015), internal axial and radial conductivity and the conductance of the shortest routes connecting the roots to the shoot base (Lobet et al., 2014), the ability of roots to explore the heterogeneous soil resources and reach the subsoil (Lynch and Wojciechowski, 2015), and root-shoot signaling (Huber et al., 2014). Another possible strategy that plants use to optimize water uptake is to modify the rhizosphere, the environment in which the roots grow and with which they interact (Hinsinger et al., 2009; York et al., 2016).

The rhizosphere is the soil around the roots; it interacts with and is altered by the roots in several ways. Root growth compacts the soil and decreases the porosity of the rhizosphere (Aravena et al., 2011). Roots shrink when they dry, creating gaps between soil and roots (Carminati et al., 2009). Cycles of repeated drying and wetting in the rhizosphere increase soil aggregation (Watt et al., 1994). Manifold studies on soil structure, soil aggregation, and water distribution around the roots have shown that the physical properties of the rhizosphere are unlike those of the bulk soil in several ways (Watt et al., 1994; Young, 1995; Carminati et al., 2010; Ahmed et al., 2016a). Of particular interest is the presence of mucilage, a polymeric gel exuded by most plant roots. Mucilage is thought to be one of the main factors influencing the rhizosphere hydraulic properties and is considered to have several functions, including maintaining good contact between roots and soil particles, promoting root penetration by reducing friction, facilitating root water uptake by increasing the water content of the rhizosphere, and preventing dehydration of root tissues as the root tip emerges (McCully, 1995; Hallett et al., 2003; Carminati et al., 2011; Ahmed et al., 2014).

Although mucilage can absorb large volumes of water (McCully and Boyer, 1997), it also contains a small fraction of amphiphilic components such as lipids (Read et al., 2003). Under dry conditions, the hydrophilic ligands of these components may bind to each other or to the soil and expose the hydrophobic parts toward the air-filled phase of the pore space, causing water repellency in the rhizosphere. Rhizosphere water repellency has been observed in barley (Hordeum vulgare L.) (Hallett et al., 2003), lupines (Carminati et al., 2010; Moradi et al., 2012; Zarebanadkouki et al., 2016), Vicia faba L. and wheat (Triticum aestivum L.) (Zickenrott et al., 2016), and maize (Ahmed et al., 2015, 2016a). Zarebanadkouki and Carminati (2014) showed that rhizosphere water repellency temporarily limited root water uptake after soil drying and subsequent rewetting. Ahmed et al. (2016a) recently proved that this rhizosphere hydrophobicity was caused by mucilage exudation.

Such advances in rhizosphere research have become possible with the recent development of noninvasive methods such as
X-ray computed tomography, nuclear magnetic resonance, twodimensional light transmission imaging, and neutron radiography (Garrigues et al., 2006; Moradi et al., 2013; Pohlmeier et al., 2013; Koebernick et al., 2014). Compared with other imaging methods, neutron radiography has proven to be one of the most useful for studying root-soil water relations (Carminati et al., 2010; Esser et al., 2010; Carminati, 2013; Kroener et al., 2015; Dara et al., 2015; Ahmed et al., 2016b). The reason for the success of this method is the high sensitivity of neutrons to materials containing a large fraction of $\mathrm{H}$, such as water.

To better illustrate the effect of mucilage on rhizosphere hydrophobicity, we refer to Fig. 1, which shows the water distribution around lupine and maize roots $30 \mathrm{~min}$ after irrigation. The images were obtained using neutron radiography. Details of the experiments were described by Zarebanadkouki et al. (2016) and Ahmed et al. (2016a), respectively. The gray values are proportional to the water content $(\mathrm{dark}=$ wet, bright $=\mathrm{dry})$. The samples were irrigated from the top at a constant flow rate of $1 \mathrm{~mL} \mathrm{~min}^{-1}$ using a peristaltic pump. Both radiographs show that although the bulk soil was rewetted, the rhizosphere remained markedly dry.

The aim of this study was to introduce a new concept to manage the rhizosphere physical and hydraulic properties to increase plant tolerance to prolonged periods of soil drying. The question is: what are the properties of an optimal rhizosphere? The first point concerns the rhizosphere hydrophobicity on drying (Fig. 1). A plant with a wettable rhizosphere would have access to a larger volume of water after irrigation. Therefore, our first target was to increase the rewetting rate of the rhizosphere following irrigation.

A second aspect concerns the hydraulic conductivity of the rhizosphere. Contrary to existing paradigms, Tardieu and Parent (2017) showed that "decreasing root hydraulic conductance when evaporative demand is maximum can improve plant performance" under conditions of water scarcity. The idea is that a low root hydraulic conductance induces an early closure of the stomata, with a consequent moderation of transpiration and slower consumption of the soil water resources. We believe that we can achieve the same effect by manipulating rhizosphere hydraulic properties. For instance, we tested whether a reduction in mucilage swelling and a consequent increase in its viscosity would reduce the hydraulic conductivity of the rhizosphere, inducing a moderate stress to the plants and decreasing transpiration. This slower water consumption would allow the plant to stay turgid during a prolonged period of soil drying, and it would be highly beneficial under climatic conditions with high evaporative demand.

We define the concept of a rhizoligand as an additive that (i) increases the rhizosphere wettability and (ii) decreases mucilage swelling and increases its viscosity. Because surfactants have been proposed as a possible mean to increase the wettability of water-repellent soils (Kostka et al., 1997; Kostka, 2000; Dekker et al., 2005), we first 


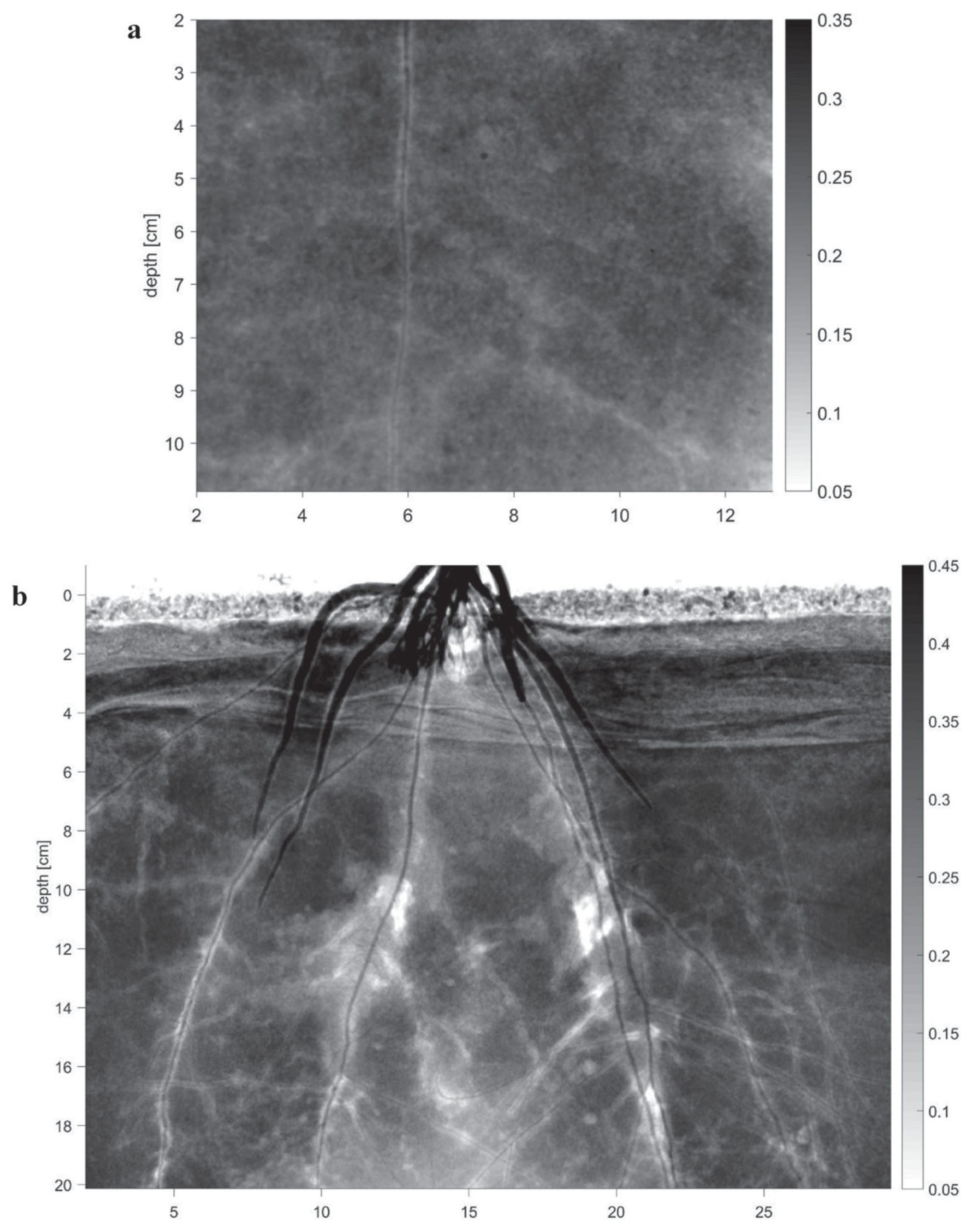

Fig. 1. Water distribution around the roots of (a) 4 wk-old lupine and (b) 4 wk-old maize plants 30 min after irrigation. The images were obtained using neutron radiography. The gray values are proportional to the water content (dark = wet, bright $=$ dry). The figure shows that the rhizosphere of neither lupine nor maize was rewetted on irrigation. The key shows the soil water content.

tested their applicability to water dynamics in the rhizosphere. As a second step, we tested if the selected surfactant behaved as a rhizoligand, and we studied its effects on transpiration.

We hypothesized the following mode of action of the interactions between a rhizoligand (in this case a surfactant) and mucilage: rhizoligands have hydrophilic and hydrophobic functional groups. When the rhizoligand concentration is high enough, the molecules of the rhizoligand rearrange with the hydrophilic head or tail attempting to increase contact with the water molecules, increasing the rehydration of mucilage and the rewetting of the rhizosphere. On the contrary, the hydrophobic head or tails of rhizoligand molecules associate with the hydrophobic groups of the mucilage network. We hypothesize that the interaction between the hydrophobic components of mucilage and a rhizoligand promote the gelation of the mucilage network by cross-linking the 
network. The cross-linking is expected to reduce mucilage swelling and increase its viscosity, reducing the hydraulic conductivity of the rhizosphere.

\section{Materials and Methods}

\section{Soil and Plant Preparation}

We used a sandy soil that was collected from the artificial catchment of Chicken Creek, located near Cottbus, Germany. The soil consisted of $92 \%$ sand, $5 \%$ silt, and approximately $3 \%$ clay. It was sieved to a particle size $<2 \mathrm{~mm}$ and then added to aluminum containers. The size of the containers was 15 by 30 by $1 \mathrm{~cm}$. The containers were deliberately constructed to a thickness of $1 \mathrm{~cm}$ as a requirement of neutron radiography. The aluminum containers were laid horizontally and the detachable top sheet was removed. The sandy soil was then poured through a sieve to minimize the formation of soil layers. The top sheet of each container was then closed, and the containers were turned vertically and gently shaken to achieve a stable packing. The resulting bulk density was $1.45 \mathrm{~g} \mathrm{~cm}^{-3}$. The aluminum containers had holes that allowed irrigation from the bottom.

Lupine and maize seeds were soaked in $10 \% \mathrm{H}_{2} \mathrm{O}_{2}$ solution for $5 \mathrm{~min}$ and then germinated on moist filter paper for $48 \mathrm{~h}$. The seedlings were then planted at a depth of $1 \mathrm{~cm}$ into the containers (one seed per container). The upper soil layers were covered with a $1-\mathrm{cm}$ layer of quartz gravel (3-mm diameter) to minimize evaporation. The plants were grown in a climate chamber with a daily light cycle of $14 \mathrm{~h}$ of light and $10 \mathrm{~h}$ of darkness, a light intensity of $500 \mu \mathrm{mol} \mathrm{m}^{-2} \mathrm{~s}^{-1}$, day and night temperatures of 24 and $19^{\circ} \mathrm{C}$, respectively, and a relative humidity of $60 \%$. During the first $2 \mathrm{wk}$, the seedlings were irrigated only with water. After $2 \mathrm{wk}$, the plants were divided into two groups: one group was irrigated with water and the other one with water treated with a rhizoligand. In this case, the rhizoligand was a surfactant (ACA3276, Aquatrols Corporation of America), which is a hydrophobically modified nonionic polyol at a concentration of $0.05 \mathrm{~mL} \mathrm{~L}^{-1}$ water.

\section{Neutron Radiography Experiments}

Because of its high sensitivity to hydrous materials, neutron imaging has been increasingly used to image water and root distributions in soil (Oswald et al., 2008; Tumlinson et al., 2008; Moradi et al., 2008; Carminati et al., 2010). In neutron radiography, a beam of neutrons is used as penetrating radiation to probe a sample. The number of neutrons passing through the sample depends on the sample thickness and the attenuation coefficients of the materials in the sample according to the Beer-Lambert law (Ahmed et al., 2016b). The neutron beam can be considered parallel at short sample detector distances. The transmitted beam is detected by a charge-coupled device (CCD) camera, and the information is converted into a digital image. The detected image carries information on the thickness and composition of the sample. Our experiments were performed at the ICON beam line of the Paul Scherrer Institute, Switzerland (Kaestner et al., 2011). We used a sCMOS camera with an array of 2160 by 2560 pixels, resulting in a field of view of 13.3 by $16 \mathrm{~cm}$ and a pixel size of $0.062 \mathrm{~mm}$.

The aim of the neutron radiography experiments was to monitor the water distribution in the rhizosphere of lupine and maize irrigated with water and a rhizoligand. The neutron radiography measurements started when the plants were $30 \mathrm{~d}$ old and had a water content of $0.04 \mathrm{~m}^{3} \mathrm{~m}^{-3}$. The water content was monitored by weighing the containers. The plants were then irrigated from the top at a constant flow rate of $1 \mathrm{~mL} \mathrm{~min}^{-1}$ using a peristaltic pump, and time-series neutron radiography was used to monitor water redistribution in the soil and roots. Half of the samples were irrigated with water and the other half with water treated with the rhizoligand at a concentration of $0.05 \mathrm{~mL} \mathrm{~L}^{-1}$.

\section{Drying and Rewetting Experiments}

The aim of this experiment was to test the effect of a rhizoligand on the transpiration rate of lupine and maize subjected to repeated drying and wetting cycles. Ten lupine and ten maize plants were grown in aluminum containers filled with the same sandy soil as used in the neutron radiograph experiment. During the first $2 \mathrm{wk}$, the plants were irrigated only with water. After $2 \mathrm{wk}$, the samples were allowed to dry. When the plants showed symptoms of wilting, they were irrigated again. Half of the samples were immersed in water and half in water treated with the rhizoligand. After $1 \mathrm{~h}$, the water at the bottom was allowed to drain and another drying cycle was repeated. During the drying cycles, we measured the transpiration rate by weighing the samples at regular intervals. In a parallel experiment, we measured the water loss by evaporation in unplanted samples.

\section{Mucilage Viscosity Measurements}

Mucilage viscosity was assessed for different mucilage concentrations. We used mucilage collected from chia seeds (Salvia hispanica L.) as a model of plant mucilage (Ahmed et al., 2014; Kroener et al., 2015). The chemical composition of chia seed mucilage is similar to that of maize: both are mainly composed of xylose, glucose, and uronic acids. In both species, the mucilage content of uronic acid is approximately 25\% (Lin et al., 1994; Carminati and Vetterlein, 2013). Furthermore, mucilage from chia seeds has a similar physical behavior to that of mucilage from maize and lupine. It has also been shown that the mucilage of chia seeds contains significant amounts of polysaccharides that form a gel-like network around the seeds (Lin et al., 1994; Muñoz et al., 2012). Indeed, when we immersed chia seeds in water, mucilage appeared immediately as a transparent capsule around the seeds. We also dried the mucilage of the chia seeds and observed a high contact angle and consequent hydrophobicity (Benard et al., 2016).

Mucilage was extracted according to the procedure described by Ahmed et al. (2014). The mucilage viscosity at 10 different mucilage 
concentrations was assessed using a viscometer (Brookfield Model LVDV-I, Brookfield Engineering Laboratories).

\section{Mucilage Swelling Experiments}

To test the effect of the rhizoligand on mucilage swelling, mucilage from chia seeds was again used as a model of plant mucilage. We measured the maximum swelling of the mucilage by immersing a given amount of dry mucilage into $6 \mathrm{~mL}$ of water or water treated with the rhizoligand. We allowed the mucilage to hydrate for $3 \mathrm{~d}$ and then removed the amount of water that was not adsorbed into the gel by pouring the gel solution through a $1-\mathrm{mm}$ sieve. The weight of the remaining solution was measured.

\section{Saturated Hydraulic Conductivity}

We measured the effect of the rhizoligand on the saturated hydraulic conductivities of soil mixed with mucilage. Mucilage was mixed with a sandy soil as described by Ahmed et al. (2014) and then was allowed to dry. Cylinders ( $2 \mathrm{~cm}$ in diameter) were filled with $10 \mathrm{~g}$ of dry mucilage-soil mixture and then saturated with water or water treated with the rhizoligand. The saturated hydraulic conductivities of the soil samples were estimated by imposing a constant difference in pressure head between the top and bottom of the sample and measuring the stationary water outflow from the samples.

\section{Modeling Root Water Uptake}

The objective of this model was to simulate how the change in the hydraulic properties of a rhizosphere treated with a rhizoligand impacts transpiration and the duration of drying cycles and to test if the simulation results reproduced the experimental observations.

The simulations were based on the model described by Carminati et al. (2016), who discussed the role of mucilage on soil-plant water relations and their potential implications for plant drought tolerance. They developed a simple model of root water uptake coupled with mucilage dynamics. In the model, the rhizosphere hydraulic properties depend on the mucilage concentration, and the root system is simplified as a single-root model. Transpiration depends on stomatal conductance, which in turn depends on the water potential at the root surface. The details of the model implementation were described by Carminati et al. (2016). Here, we give a brief description of the model.

The water flow in the soil was simulated solving the Richards equation in radial coordinates:

$$
\frac{\partial \theta}{\partial t}=\frac{1}{r} \frac{\partial}{\partial r}\left(r \frac{\partial h}{\partial r}\right)
$$

where $t$ is time and $r$ is the radial coordinate. This equation was solved analytically using the steady-rate assumption as used by Carminati et al. (2011). The outer soil domain was set to $1 \mathrm{~cm}$. The outer boundary condition was no flow. The inner boundary condition was a variable flow, $q_{\text {act }}\left(\mathrm{cm} \mathrm{s}^{-1}\right)$, that depended on the matric potential at the root surface, $h_{0}$, according to

$q_{\text {act }}=q_{\text {pot }}\left[1-\exp \left(\frac{h_{0}-15,000}{h_{2}}\right)\right]$

where $q_{\text {pot }}\left(\mathrm{cm} \mathrm{s}^{-1}\right)$ is the maximum flow at the root surface and $h_{2}(\mathrm{~cm})$ is a fitting parameter set to 300 . The value of $q_{\text {pot }}$ and the root length were chosen to fit the total soil volume and water losses from the sample. According to Eq. [2], the flux is constant for high matric potentials and then it gradually decreases as the matric potential becomes more negative. The soil hydraulic properties were those of a sandy soil and were reported by Carminati et al. (2016).

\section{Results}

\section{Rhizosphere Rewetting: Water Distribution around Lupine Roots observed with Neutron Radiography}

We used neutron radiography to image the soil water distribution in samples planted with lupines that underwent a drying cycle and were subsequently irrigated with water or water treated with a rhizoligand. Figure 2 shows the water content distribution in the root zone of 30-d-old lupine $30 \mathrm{~min}$ after irrigation. Before irrigation, the water content in the two samples was 0.02 to $0.04 \mathrm{~cm}^{3} \mathrm{~cm}^{-3}$. In the images, the water content is proportional to the gray values, i.e., darker values are wetter. Figure 2 (left) shows a sample irrigated with water. In the image, the rhizosphere of most roots appears brighter than the bulk soil. This shows that the rhizosphere remained dry after irrigation. However, when irrigated with the rhizoligand solution (Fig. 2, right) the rhizosphere appears as dark as the bulk soil, indicating that the rhizosphere was quickly rewetted. We conclude that the rhizoligand facilitated the wetting rate of the rhizosphere. The rhizosphere of the lupine irrigated with the rhizoligand rewetted within minutes. The experiment was replicated five times (five samples irrigated with water and five with rhizoligand). All samples showed the same behavior.

\section{Effect of Rhizoligand on Soil Water Availability during Drying-Wetting Cycles}

In this experiment, we tested the effects of the rhizoligand on the root water uptake of lupine and maize during repeated drying and wetting cycles. The soil water content, $\theta$ (volume of water divided by the total soil volume), during one drying cycle is shown in Fig. 3. The figure shows the water content after irrigation and until the plants began to wilt. The first points correspond to $4 \mathrm{~h}$ after irrigation. Plant irrigated with the rhizoligand (red) started with a higher water content than the plants irrigated with water. The higher water content in the rhizoligand-irrigated treatment was the result of the rhizoligand rewetting the rhizosphere (as shown in Fig. 2, right). On the 

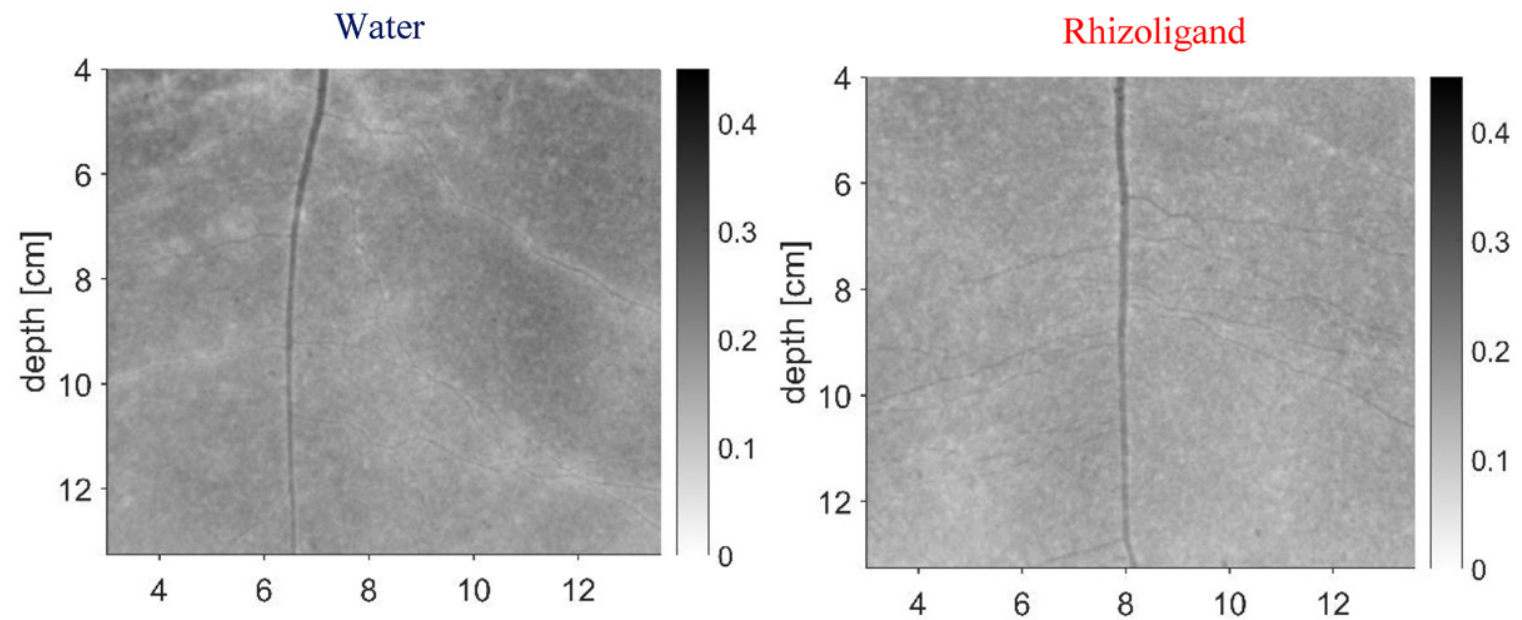

Fig. 2. Water distribution around the roots of 30-d-old lupines $30 \mathrm{~min}$ after irrigation. The images were obtained using neutron radiography. Dark colors indicate high water contents, bright colors low water contents. The rhizosphere of the sample irrigated with water stayed dry after irrigation (left). The rhizosphere of the sample irrigated with the rhizoligand quickly rewetted after irrigation (right).

contrary, in the samples irrigated with water (blue), the rhizosphere remained dry. Plants irrigated with the rhizoligand also wilted $2 \mathrm{~d}$ later than those irrigated with water. Interestingly, the curves of the water and rhizoligand treatments had a similar slope prior to a "breaking point" that occurred at approximately $60 \mathrm{~h}$ after irrigation. The fact that the slope of the rhizoligand treatment (red) is lower than the slope of the water treatment (blue) at approximately $\theta=0.05$ suggests that the effect of the rhizoligand on root water uptake was actually more pronounced at low soil water contents.

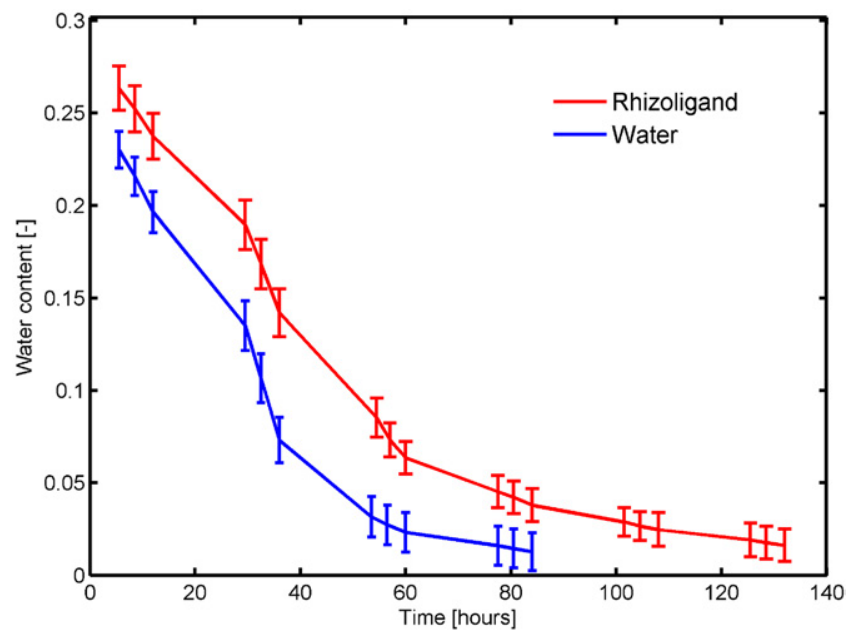

Fig. 3. Average water content $(\theta)$ in the lupine samples irrigated with water (blue, $n=5$ ) and water treated with a rhizoligand (red, $n=5$ ) during the last drying cycle of the experiment. Time refers to the time after irrigation. The plant irrigated with the rhizoligand started with a higher water content. The increased water content in the samples irrigated with the rhizoligand was caused by the fact that the rhizoligand rewetted the rhizosphere (as shown in Fig. 2), while in the samples irrigated with water, the rhizosphere remained dry. The plants irrigated with the rhizoligand also wilted $2 \mathrm{~d}$ later than those irrigated with water.
The transpiration rate was derived from the change in water content. Transpiration rates during the same drying cycle are plotted in Fig. 4 . The first points correspond to $4 \mathrm{~h}$ after irrigation. Figure 4 shows that in both treatments, the transpiration rate increased for 1 to $2 \mathrm{~d}$ after irrigation. The increasing-transpiration phase corresponded to the plant recovery and reopening of stomata after severe drying. During this phase, the transpiration rate in the plants irrigated with water was higher than in the plants irrigated with the rhizoligand. Approximately $40 \mathrm{~h}$ after irrigation, the transpiration rate began to decrease due to the reduction in

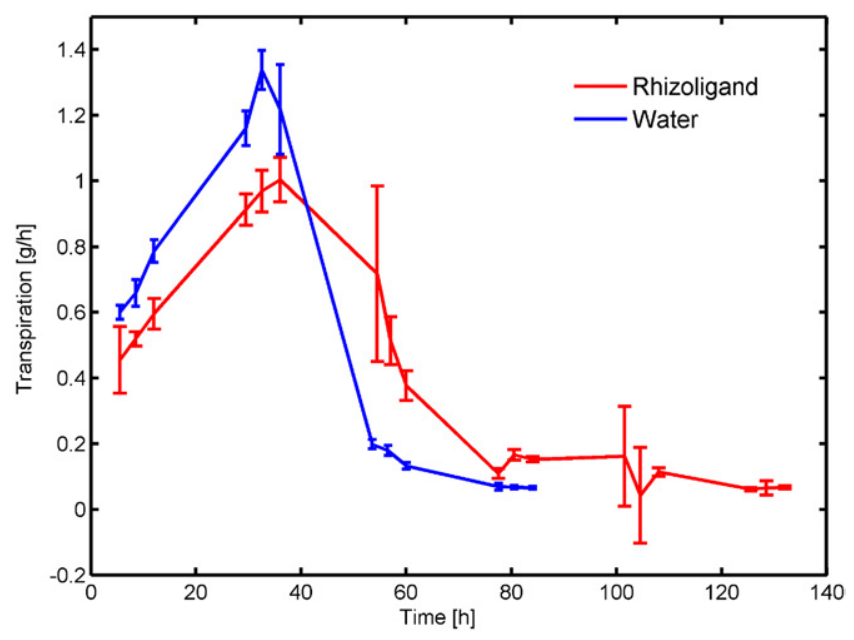

Fig. 4. Transpiration rate calculated gravimetrically during the drying phase shown in Fig. 3. The first points correspond to $4 \mathrm{~h}$ after irrigation. In both the samples irrigated with water (blue) and those irrigated with water treated with rhizoligand (red), the transpiration rate increased for 1 to $2 \mathrm{~d}$ after irrigation. This increasing-transpiration phase corresponds to the plant recovery after severe drying. During this phase, the transpiration rate in the samples irrigated with water was higher than in the samples irrigated with the rhizoligand. The transpiration rate started to decrease around $40 \mathrm{~h}$ after irrigation because of the reduction in soil water content. 
soil water content. This point is better illustrated in Fig. 5, where transpiration rates are plotted as a function of the water content.

Figure 5 shows the transpiration vs. water content during the same drying cycle. During the initial phase, when the water content was relatively high, transpiration increased with decreasing water content. This could be explained by the plant recovery and reopening of stomata after drying and subsequent irrigation. Figure 5 shows that, with time, the transpiration rate first increased to a maximum value and then started to decrease as the soil became dry. The water content at which transpiration began to decrease differed between the rhizoligand and water treatments. In the samples irrigated with water, transpiration decreased at a water content of approximately $0.1 \mathrm{~cm}^{3} \mathrm{~cm}^{-3}$. Instead, in the samples irrigated with the rhizoligand, transpiration started to decrease at a water content of approximately $0.15 \mathrm{~cm}^{3} \mathrm{~cm}^{-3}$. Although the temporal resolution of the measurement is low, we could observe that at a water content of $0.07 \mathrm{~cm}^{3} \mathrm{~cm}^{-3}$, the transpiration rate in the samples irrigated with the rhizoligand was half that in the samples irrigated with water. Plants irrigated with the rhizoligand reached the lower transpiration rate at a water content of $0.05 \mathrm{~cm}^{3} \mathrm{~cm}^{-3}$ and remained turgid until the water content dropped to 0.02 to $0.03 \mathrm{~cm}^{3} \mathrm{~cm}^{-3}$. The low transpiration rate and the initially higher water contents are the primary reasons why the rhizoligand-treated plants wilted later.

These results were obtained assuming that transpiration could be derived by weight losses in the samples. To do so, we made the following assumptions: (i) evaporation was much smaller than transpiration; and (ii) plant growth during the drying cycles was much smaller than the water losses in the soil. Our experiments with unplanted samples showed that evaporation was much smaller than transpiration (see Supplemental Fig. S1). Because the samples were small and the drying cycles were fast $(4-5 \mathrm{~d})$, the changes in plant fresh weight were also small compared with the water losses.

We then repeated the experiments but with the soil remaining wet. Ten lupines were grown under the same conditions as in the experiments described above. The plants were irrigated with water for $2 \mathrm{wk}$. After $2 \mathrm{wk}$, the plants were divided into two groups and we began irrigating the samples with either water or rhizoligand solution. Every day at 7:00 AM, we added the amount of water or rhizoligand solution that was lost by evapotranspiration during the previous day. Water or rhizoligand solution (at a concentration of $0.05 \mathrm{~mL} \mathrm{~L}^{-1}$ water) were applied to the surface of the soil. In this way, the soil water was maintained between 0.20 and 0.15 $\mathrm{cm}^{3} \mathrm{~cm}^{-3}$. At the end of the day, soils in the samples irrigated with the rhizoligand were slightly drier than the samples irrigated with water (data not shown). This means that the plants irrigated with the rhizoligand took up slightly more water. We conclude that when the samples were kept relatively wet, transpiration was not reduced by the rhizoligand. Note that the total amount of rhizoligand applied to these samples was as high as the rhizoligand

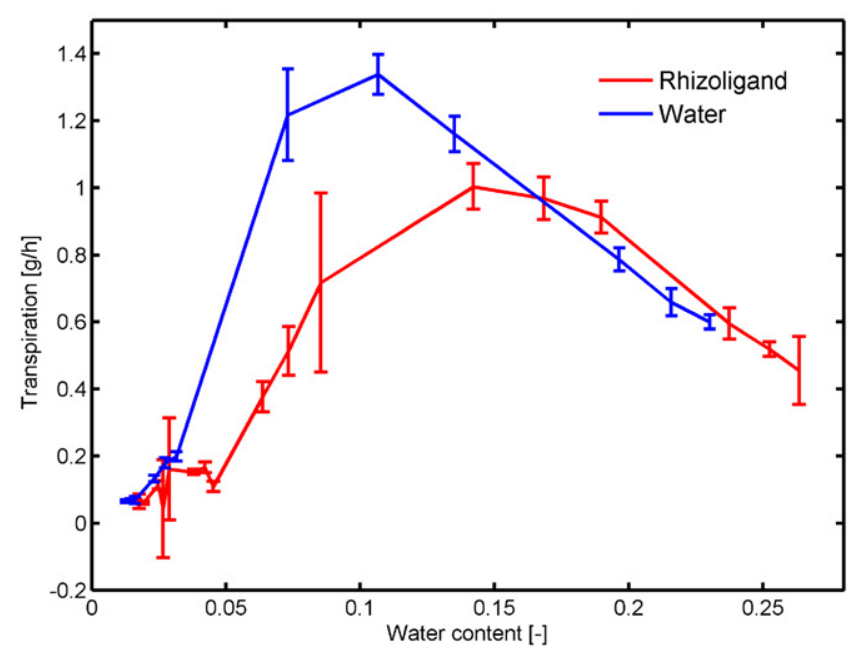

Fig. 5. Transpiration rate as a function of water content for the drying cycle shown in Fig. 3. During the initial phase when the water content was relatively high, transpiration increased with decreasing water content. When the soil became dry, transpiration started to decrease. The water content at which transpiration started to decrease was different for the two treatments. In the samples irrigated with water, transpiration decreased at a water content of approximately 0.1 . Conversely, in the samples irrigated with the rhizoligand, transpiration had already begun to decrease at a water content of approximately 0.15 . At a water content of 0.07 , the transpiration rate in the samples irrigated with the rhizoligand was half that of the samples irrigated with water.

applied in the samples that were allowed to dry to a lower content. Parallel experiments with unplanted samples showed that water losses by evaporation were negligible compared with transpiration and were equal in the two treatments (see Supplemental Fig. S1).

We also tested the effects of the rhizoligand on the root water uptake of maize during repeated drying-wetting cycles (data not shown). The responses paralleled our results with lupine. Plants irrigated with the rhizoligand started with a higher water content than the plants irrigated with water. The increased water content in the plants irrigated with the rhizoligand was caused by rhizoligand-enhanced rewetting of the rhizosphere, whereas in the treatment irrigated with water, the rhizosphere remained dry (Fig. 1b). As a consequence of enhanced rhizosphere rewetting, the plants irrigated with the rhizoligand wilted later than those irrigated with water. An assessment of transpiration with time showed that transpiration curves for maize followed similar patterns to those for lupines. Transpiration rates in maize were also reduced after repeated drying-wetting cycles.

\section{Mucilage Viscosity Measurements}

The aim of this experiment was to measure the viscosity of different mucilage concentrations. Figure 6 shows the viscosity of 10 different mucilage concentrations. The mucilage viscosity increased with increasing mucilage concentration. The mucilage viscosity decreased with increasing rotational velocity, which is typical of gels showing a shear thinning behavior. The consequence of the shear thinning behavior is that the hydraulic conductivity of 


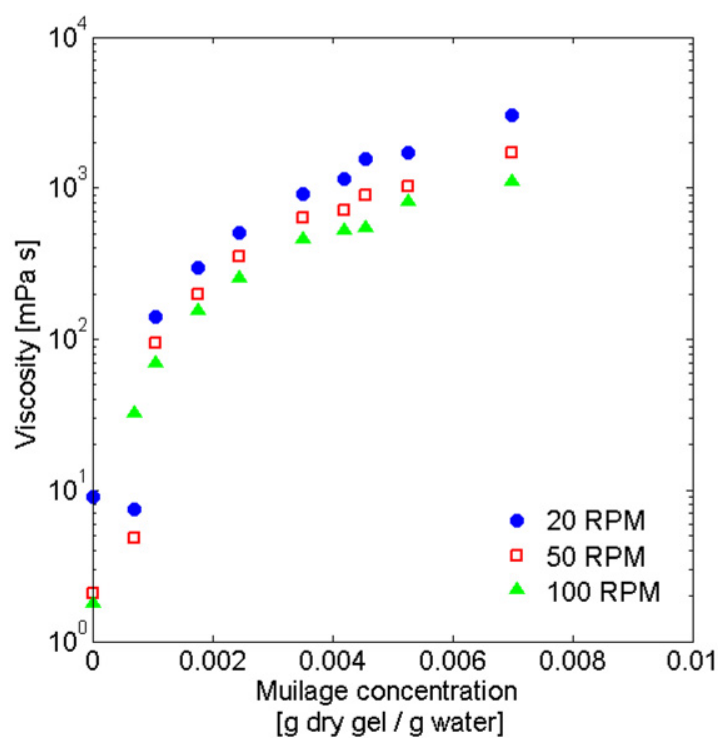

Fig. 6. Mucilage viscosity as a function of mucilage concentration. The mucilage viscosity increased by three orders of magnitude with increasing mucilage concentration. Mucilage viscosity decreased with increasing rotational velocity, which is typical of a gel showing a shear thinning behavior.

soils mixed with mucilage might increase with water fluxes, which would have important consequences for enabling high water fluxes across the rhizosphere.

\section{Effect of Rhizoligand on Mucilage-Soil Water Relations}

Our hypothesis was that the suppression of transpiration when plants were irrigated with a rhizoligand was related to the hydraulics of the rhizosphere. This hypothesis was based on the effects of the rhizoligand on the maximum swelling and viscosity of gels containing hydrophobic components (Simovic et al., 1999). Simovic et al. (1999) showed that, at specific concentrations, nonionic surfactants decrease the swelling and increase the viscosity of gels containing hydrophobic components. It is important to note that mucilage exuded by roots contains lipids (Read et al., 2003).

The effect of a rhizoligand on mucilage was evaluated using mucilage from chia seeds. Mucilage from chia seeds has similar physical and chemical properties to that of mucilage from maize (Ahmed et al., 2014). We measured the maximum swelling of the mucilage by immersing a given amount of dry mucilage in $6 \mathrm{~mL}$ of water or water treated with the rhizoligand. The rhizoligand treatment significantly decreased the maximum swelling of the mucilage (Fig. 7). Note that some degree of mucilage drying is needed to trigger the effect of the rhizoligand. In fact, when a rhizoligand infiltrates through the network of dry and shrunk mucilage, it binds the elements of the network and impedes its swelling. This effect does not occur when the rhizoligand is applied to hydrated and swollen mucilage. Such results of mucilage swelling are consistent with the effects of the rhizoligand on transpiration, i.e., the rhizoligand decreased transpiration only when a drying cycle was applied.

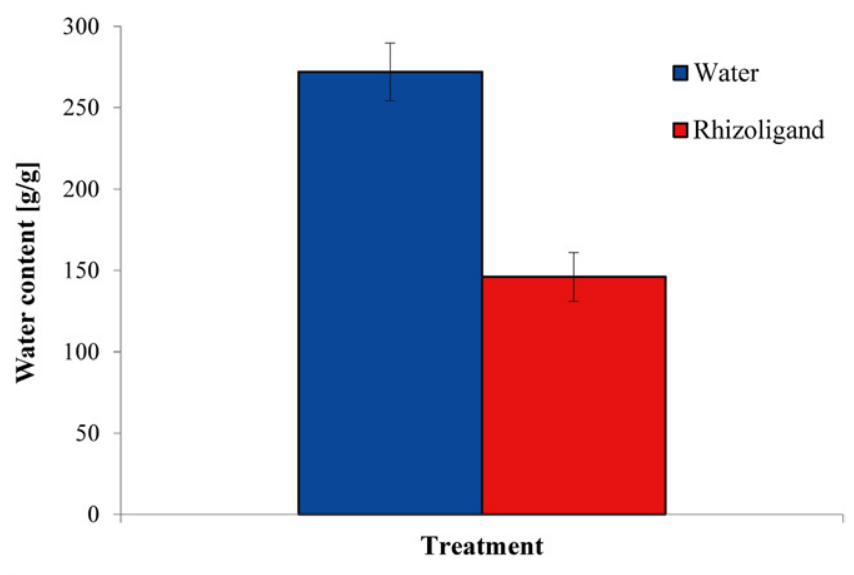

Fig. 7. Saturated water content of mucilage in water and water treated with a rhizoligand. The rhizoligand significantly decreased the maximum swelling of the mucilage. Data represent mean $\pm \operatorname{SD}(n=7)$.

\section{Saturated Hydraulic Conductivity}

The aim of this experiment was to measure the effect of the rhizoligand on the saturated hydraulic conductivities of soil mixed with different mucilage concentrations. We found that mucilage decreased the saturated hydraulic conductivity of the soil, especially at high mucilage concentrations (Fig. 8). We also found that rhizoligand treatment further decreased the saturated hydraulic conductivity of soil mixed with mucilage.

\section{Modeling Root Water Uptake}

Our calculations show that a reduction by 30 times of the rhizosphere hydraulic conductivity reproduced well the decrease in transpiration (Fig. 9). The lower rhizosphere hydraulic conductivity induced an early closure of the stomata, with a consequent moderation of transpiration and slower consumption of the soil water. The imposed reduction of 30 times is higher than

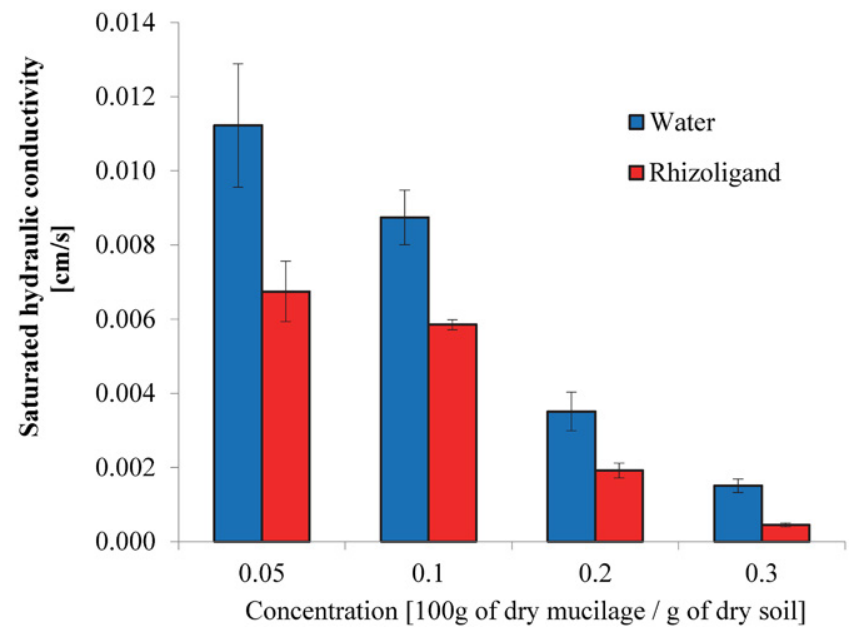

Fig. 8. Saturated hydraulic conductivity of soils mixed with varying mucilage concentrations. The samples were saturated with water (blue) and water treated with a rhizoligand (red). The rhizoligand decreased the saturated hydraulic conductivity of the soil. Data represent mean $\pm \mathrm{SD}(n=4)$. 

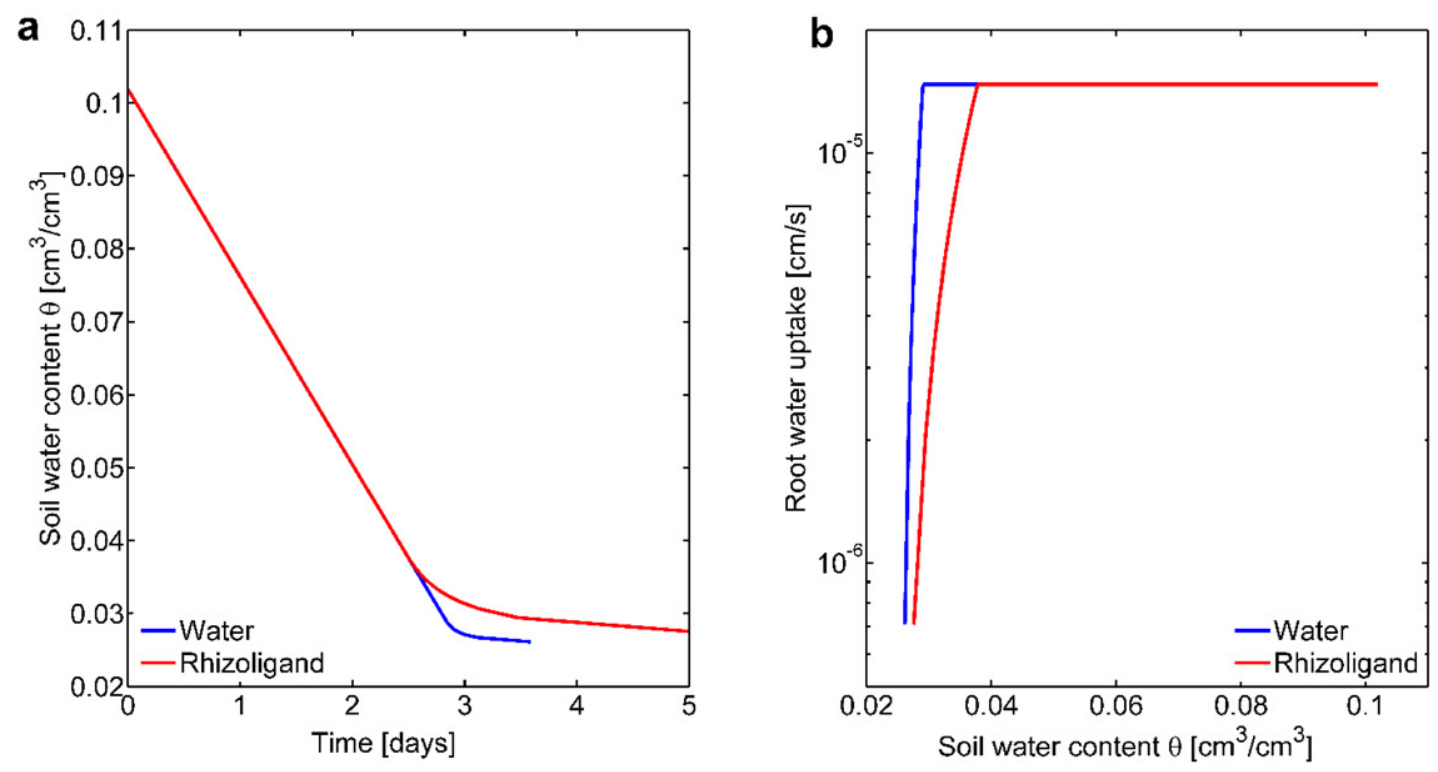

Fig. 9. Effect of a rhizoligand on soil water content and root water uptake during a drying period: (a) soil water content during one drying cycle for irrigation with water (blue) and water treated with a rhizoligand (red); and (b) root water uptake as a function of soil water content. We assumed that the rhizoligand reduced the rhizosphere hydraulic conductivity by 30 times, which resulted in an earlier decrease in transpiration. The simulations reproduce well the experimental observations.

the observed decrease in conductivity (Fig. 8). The choice was motivated by the fact that the plants were exposed to repeated rhizoligand applications and drying-wetting cycles so that the effects on the plants were probably stronger than in the samples used to measure the saturated conductivity. The simulations reproduced well the decreased transpiration at low water contents. In this study, we used this model to test the hypothesis that a decreased hydraulic conductivity of the rhizosphere decreases transpiration. The goal was not to fit the experimental observation but rather to qualitatively confirm our new concept.

\section{Discussion}

We propose a method to manage the wetting dynamics and hydraulic properties of the rhizosphere. The tested surfactant behaved as a rhizoligand: it increased the wetting kinetics of the rhizosphere, as well as the uniformity of rhizosphere rewetting. The consequence was an increase in the volume of water available to the plant. Remarkably, the increased water volume was also consumed more slowly because transpiration was suppressed in plants with rhizoligand-treated soils.

To better illustrate the effect of a rhizoligand on rhizosphere rewetting and root water uptake, we developed a conceptual model of water flow through a soil mixed with mucilage that was initially dry and then irrigated with water and a rhizoligand (Fig. 10). This model is applicable to a dry rhizosphere that is subsequently irrigated. Rhizoligand treatment decreases the contact angle and facilitates the initial rewetting of the soil-mucilage mixture compared with soil irrigated with water (short time effect). This faster rhizosphere rewetting probably facilitates the rehydration of the root tissues; however, this is not reflected in a faster increase in transpiration. With time, the mucilage rehydrates and water flow depends on the mucilage swelling. Rhizoligands decrease mucilage Time after irrigation

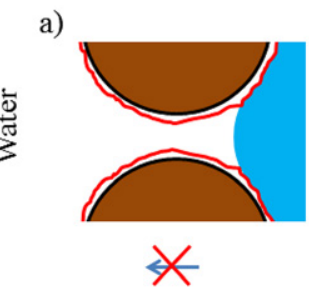

b)
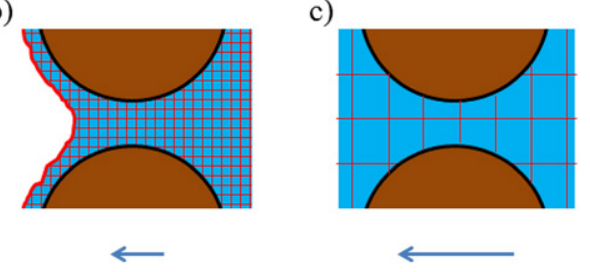

d)

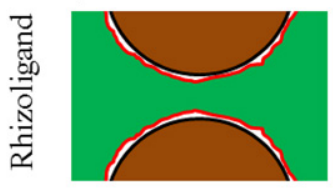

e)

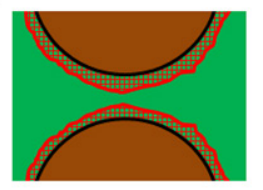

f)

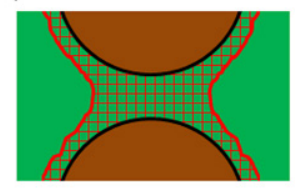

Fig. 10. Conceptual model of infiltration of water and a rhizoligand in soils covered with dry mucilage. The pore space between two soil particles covered with dry mucilage is illustrated during rewetting: (a) dry mucilage is hydrophobic and temporarily limits the soil rewetting; (b) as mucilage starts to adsorb water, the soil rewets; (c) the soil hydraulic conductivity increases as the gel hydrates; (d) the rhizoligand reduces the contact angle and rewets the soil; (e) as the gel swells, the soil hydraulic conductivity decreases; and (f) because the rhizoligand decreases mucilage swelling, the mucilage does not fully expand, resulting in a reduced soil hydraulic conductivity. 
swelling and therefore decrease the saturated conductivity of the soil-mucilage mixture (long-term effect).

Our explanation of the reduction in transpiration in plants growing in soils treated with rhizoligands is related to the rhizoligands' interactions with mucilage. Mucilage has a crucial role in maintaining the hydraulic connection between soil and roots (Carminati et al., 2010). By modifying mucilage swelling, rhizoligands affect the hydraulic conductivity of the root-soil interface. Specifically, rhizoligands decrease mucilage swelling after drying and therefore limit the diffusion of mucilage far from the roots. The suppressed mucilage swelling results in a decreased hydraulic conductivity of the rhizosphere, which possibly explains the lower transpiration of the plants irrigated with a rhizoligand. The fact that transpiration was not reduced in the plants that were kept wet can also be related to mucilage dynamics. In fact, when soils were kept wet, the mucilage remained hydrated and its network was swollen. We expect that when mucilage is highly hydrated, rhizoligands cannot reduce its swelling. On the contrary, some degree of mucilage drying is needed for the rhizoligands to be able to cross-link the polymers in the mucilage.

Carminati et al. (2016) discussed the role of mucilage on soil-plant water relations and its potential implications for plant drought tolerance. Thy developed a simple model of root water uptake coupled with mucilage dynamics. In the model, the rhizosphere hydraulic properties depend on the mucilage concentration, and the root system is simplified as a single-root model. Transpiration depends on stomatal conductance, which in turn depends on the water potential at the root surface. In this study, we used this model to test the hypothesis that a decreased hydraulic conductivity of the rhizosphere decreases transpiration. The calculations show that a reduction by 30 times of the rhizosphere hydraulic conductivity reproduced well the decrease in transpiration (Fig. 9). The lower rhizosphere hydraulic conductivity induced an early closure of the stomata, with a consequent moderation of transpiration and slower consumption of the soil water. The imposed reduction of 30 times is higher than the observed decrease in conductivity (Fig. 8). The choice was motivated by the fact that the plants were exposed to repeated rhizoligand applications and drying-wetting cycles, so that the effects on the plants were probably stronger than in the samples used to measure the saturated conductivity.

A second reason of the longer drying period after rhizoligand application is the slower recovery of transpiration subsequent to irrigation with the rhizoligand (Fig. 4-5). Opening and closing of stomata during drying-wetting cycles depends on both physical and chemical signals (Dodd et al., 2015). Rhizoligand treatment increased the wettability of the rhizosphere, but this was not reflected in a quicker reopening of the stomata. One hypothesis is that surfactants might have entered the xylem, inducing some embolism that limited the recovery of transpiration. An alternative hypothesis is that the lower transpiration following irrigation with the rhizoligand was caused by a quick pulse of plant hormone abscisic acid (ABA) from the roots to the shoot. There is a large body of evidence suggesting that ABA is one of the components involved in the control of stomatal conductance as the soil dries (Blackman and Davies, 1985; Davies and Zhang, 1991; Dodd, 2009; Dodd et al., 2015). We suggest that the rhizoligand rewets the rhizosphere of the driest root segments and induces a convective flux of water that transports ABA to the xylem and to the shoots, where it temporarily inhibits transpiration. In the samples irrigated with water, the rhizosphere water repellency limits the radial flux from the soil into the roots, and the ABA accumulated in these roots is not transported to the shoot. Moreover, the fact that transpiration was not suppressed by the rhizoligand when the samples were kept wet further supports this notion. In fact, the ABA pulse liberated by the rhizoligand required soil drying and rhizosphere water repellency.

We do not exclude the possibility that other mechanisms contributed to the suppression of transpiration. For instance, if a rhizoligand (surfactant) enters into the plants, it may reduce the surface tension in the xylem and either induce cavitation or retard the refilling of the xylem after embolism. The presence of a rhizoligand in the stomatal cavity may also reduce the pressure jump between the water and air and reduce the suction needed to pull water from the soil. Further studies are needed to explain what mechanisms are primarily responsible for the reduction in transpiration.

The concept of improving drought tolerance by reducing the hydraulic conductivity of the soil-root continuum, which seems counterintuitive, was recently explained by Tardieu and Parent (2017). They showed that unexpected behaviors emerge from short-term feedbacks among root water uptake, stomatal conductance, leaf elongation, and water balance. For example, "decreasing root hydraulic conductance when evaporative demand is maximum can improve plant performance." The same result was found in the numerical simulations of Couvreur et al. (2014), who showed that a lower root conductivity increased the collar xylem potential during prolonged periods of soil drying. We believe that we can achieve the same effect by manipulating rhizosphere hydraulic properties. This slower water consumption would allow the plant to remain turgid during a prolonged period of soil drying, which would be highly beneficial under climatic conditions with high evaporative demand.

In conclusion, we found that a rhizoligand improved the drought tolerance of crop plants during repeated drying-wetting cycles. We used a commercial surfactant, but the concept of a rhizoligand might not apply to all surfactants and is not limited to them. The improved drought tolerance resulted from (i) a better rewetting of the rhizosphere and (ii) a reduction in transpiration. The first point is fundamental in coarse-textured soils, which are more susceptible to water repellency, and in shallow-rooted plants, which have the largest fraction of the roots in the topsoil. An example of 
such a system is turfgrass, where surfactants have frequent commercial application (Kostka, 2000). The second point (reduced transpiration) is new and its implications are not well known. In our view, the reduced transpiration effect has applications to irrigated agriculture in regions with limited water availability, and it can be used to trigger the closure of stomata and increase the water use efficiency of irrigated crops.

\section{Acknowledgments}

We are grateful to the staff at the ICON imaging station of the Paul Scherrer Institute (PSI) for their technical support during the measurements with neutron radiography. The position of Mutez Ahmed was funded by DAAD. The research was supported by Aquatrols Corporation of America.

\section{References}

Ahmed, M.A., M. Holz, S.K. Woche, J. Bachmann, and A. Carminati. 2015. Effect of soil drying on mucilage exudation and its water repellency: A new method to collect mucilage. J. Plant Nutr. Soil Sci. 178:821-824. doi:10.1002/jpln.201500177

Ahmed, M.A., E. Kroener, P. Benard, M. Zarebanadkouki, A. Kaestner, and A. Carminati. 2016a. Drying of mucilage causes water repellency in the rhizosphere of maize: Measurements and modelling. Plant Soil 407:161-171. doi:10.1007/s11104-015-2749-1

Ahmed, M.A., E. Kroener, M. Holz, M. Zarebanadkouki, and A. Carminati. 2014. Mucilage exudation facilitates root water uptake in dry soils Funct. Plant Biol. 41:1129-1137. doi:10.1071/FP13330

Ahmed, M.A., M. Zarebanadkouki, A. Kaestner, and A. Carminati. 2016b. Measurements of water uptake of maize roots: The key function of lateral roots. Plant Soil 398:59-77. doi:10.1007/s 111 104-015-2639-6

Aravena, J.E., M. Berli, T.A. Ghezzehei, and S.W. Tyler. 2011. Effects of root-induced compaction on rhizosphere hydraulic properties: X-ray microtomography imaging and numerical simulations. Environ. Sci. Technol. 45:425-431. doi:10.1021/es102566j

Benard, P., E. Kroener, P. Vontobel, A. Kaestner, and A. Carminati. 2016. Water percolation through the root-soil interface. Adv. Water Resour. 95:190-198.

Blackman, P.G., and W.J. Davies. 1985. Root to shoot communication in maize plants of the effects of soil drying. J. Exp. Bot. 36:39-48. doi:10.1093/jxb/36.1.39

Carminati, A. 2013. Rhizosphere wettability decreases with root age: A problem or a strategy to increase water uptake of young roots? Front. Plant Sci. 4:298.

Carminati, A., E. Kroener, M.A. Ahmed, M. Zarebanadkouki, M. Holz, and T. Ghezzehei. 2016. Water for carbon, carbon for water. Vadose Zone J. 15(2). doi:10.2136/vzj2015.04.0060

Carminati, A., A.B. Moradi, D. Vetterlein, P. Vontobel, E. Lehmann, U. Weller, et al. 2010. Dynamics of soil water content in the rhizosphere. Plant Soil 332:163-176. doi:10.1007/s $11104-010-0283-8$

Carminati, A., C.L. Schneider, A.B. Moradi, M. Zarebanadkouki, D. Vetterlein, H.-J. Vogel, et al. 2011. How the rhizosphere may favor water availability to roots. Vadose Zone J. 10:988-998. doi:10.2136/vzj2010.0113

Carminati, A., and D. Vetterlein. 2013. Plasticity of rhizosphere hydraulic properties as a key for efficient utilization of scarce resources. Ann. Bot. 112:277-290. doi:10.1093/aob/mcs262

Carminati, A., D. Vetterlein, U. Weller, H.-J. Vogel, and S.E. Oswald. 2009. When roots lose contact. Vadose Zone J. 8:805-809. doi:10.2136/vzj2008.0147

Couvreur, V., J. Vanderborght, X. Draye, and M. Javaux. 2014. Dynamic aspects of soil water availability for isohydric plants: Focus on root hydraulic resistances. Water Resour. Res. 50:8891-8906. doi:10.1002/2014WR015608

Dara, A., B.A. Moradi, P. Vontobel, and S.E. Oswald. 2015. Mapping compensating root water uptake in heterogeneous soil conditions via neutron radiography. Plant Soil 397:273-287. doi:10.1007/s $11104-015-2613-3$

Davies, W.J., and J. Zhang. 1991. Root signals and the regulation of growth and development of plants in drying soil. Annu. Rev. Plant Physiol. Plant Mol. Biol. 42:55-76. doi:10.1 146/annurev.pp.42.060191.000415
Dekker, L.W., K. Oostindie, S.J. Kostka, and C.J. Ritsema. 2005. Effects of surfactant treatments on the wettability of a water repellent grasscovered dune sand. Soil Res. 43:383-395. doi:10.1071/SR04090

Dodd, I.C. 2009. Rhizosphere manipulations to maximize "crop per drop" during deficit irrigation. J. Exp. Bot. 60:2454-2459. doi:10.1093/jxb/erp192

Dodd, I.C., J. Puértolas, K. Huber, J.G. Pérez-Pérez, H.R. Wright, and M.S.A. Blackwell. 2015. The importance of soil drying and re-wetting in crop phytohormonal and nutritional responses to deficit irrigation. J. Exp. Bot. 66:2239-2252. doi:10.1093/jxb/eru532

Esser, H.G., A. Carminati, P. Vontobel, E.H. Lehmann, and S.E. Oswald. 2010. Neutron radiography and tomography of water distribution in the root zone. J. Plant Nutr. Soil Sci. 173:757-764. doi:10.1002/jpln.200900188

Garrigues, E., C. Doussan, and A. Pierret. 2006. Water uptake by plant roots: I. Formation and propagation of a water extraction front in mature root systems as evidenced by $2 \mathrm{D}$ light transmission imaging. Plant Soil 283:83-98. doi:10.1007/s 11 104-004-7903-0

Hallett, P.D., D.C. Gordon, and A.G. Bengough. 2003. Plant influence on rhizosphere hydraulic properties: Direct measurements using a miniaturized infiltrometer. New Phytol. 157:597-603. doi:10.1046/j.1469-8137.2003.00690.x

Hinsinger, P., A.G. Bengough, D. Vetterlein, and I.M. Young. 2009. Rhizosphere: Biophysics, biogeochemistry and ecological relevance. Plant Soil 321:117-152. doi:10.1007/s 111 104-008-9885-9

Huber, K., J. Vanderborght, M. Javaux, N. Schröder, I.C. Dodd, and $\mathrm{H}$. Vereecken. 2014. Modelling the impact of heterogeneous rootzone water distribution on the regulation of transpiration by hormone transport and/or hydraulic pressures. Plant Soil 384:93-112. doi:10.1007/s $11104-014-2188-4$

Kaestner, A.P., S. Hartmann, G. Kühne, G. Frei, C. Grünzweig, L. Josic, et al. 2011. The ICON beamline: A facility for cold neutron imaging at SINQ. Nucl. Instrum. Methods Phys. Res., Sect. A 659:387-393. doi:10.1016/j.nima.2011.08.022

Koebernick, N., U. Weller, K. Huber, S. Schlüter, H.-J. Vogel, R. Jahn, et al. 2014. In situ visualization and quantification of three-dimensional root system architecture and growth using $X$-ray computed tomography. Vadose Zone J. 13(8). doi:10.2136/vzj2014.03.0024

Kostka, S.J. 2000. Amelioration of water repellency in highly managed soils and the enhancement of turfgrass performance through the systematic application of surfactants. J. Hydrol. 231-232:359-368. doi:10.1016/S0022-1694(00)00208-0

Kostka, S.J., J.L. Cisar, J.R. Short, and S. Mane. 1997. Evaluation of soil surfactants for the management of soil water repellency in turfgrass. Int. Turfgrass Soc. Res. J. 8:485-494.

Kroener, E., M.A. Ahmed, and A. Carminati. 2015. Roots at the percolation threshold. Phys. Rev. E 91(4):042706. doi:10.1103/PhysRevE.91.042706

Lin, K.J., J.R. Daniel, and R.L. Whistler. 1994. Structure of chia seeds polysaccharide exudate. Carbohydr. Polym. 23:13-18. doi:10.1016/0144-8617(94) 90085-X

Lobet, G., V. Couvreur, F. Meunier, M. Javaux, and X. Draye. 2014. Plant water uptake in drying soils. Plant Physiol. 164:1619-1627. doi:10.1104/pp.113.233486

Lynch, J.P., and K.M. Brown. 2012. New roots for agriculture: Exploiting the root phenome. Philos. Trans. R. Soc. London Ser. B 367:1598-1604. doi:10.1098/rstb.2011.0243

Lynch, J.P., and T. Wojciechowski. 2015. Opportunities and challenges in the subsoil: Pathways to deeper rooted crops. J. Exp. Bot. 66:21992210. doi:10.1093/jxb/eru508

McCully, M. 1995. How do real roots work? Some new views of root structure. Plant Physiol. 109:1-6. doi:10.1104/pp.109.1.1

McCully, M.E., and J.S. Boyer. 1997. The expansion of maize root-cap mucilage during hydration: 3. Changes in water potential and water content. Physiol. Plant. 99:169-177. doi:10.1111/j.1399-3054.1997.tb03445.x

Moradi, A.B., A. Carminati, A. Lamparter, S.K. Woche, J. Bachmann, D. Vetterlein, et al. 2012. Is the rhizosphere temporarily water repellent? Vadose Zone J. 11 (3).

Moradi, A.B., H.M. Conesa, B. Robinson, E. Lehmann, G. Kuehne, A. Kaestner, et al. 2008. Neutron radiography as a tool for revealing root development in soil: Capabilities and limitations. Plant Soil 318:243255. doi:10.1007/s 111 104-008-9834-7

Moradi, A.B., J.W. Hopmans, S.E. Oswald, M. Menon, A. Carminati, E. Lehmann, et al. 2013. Applications of neutron imaging in soil-waterroot systems In: S.H. Anderson and J.W. Hopmans, editors, Soil-waterroot processes: Advances in tomography and imaging. SSSA Spec. Publ. 61. SSSA, Madison, WI. p. 113-136. 
Muñoz, L.A., A. Cobos, O. Diaz, and J.M. Aguilera. 2012. Chia seeds: Microstructure, mucilage extraction and hydration. J. Food Eng. 108:216-224. doi:10.1016/j.jfoodeng.2011.06.037

Oswald, S.E., M. Menon, A. Carminati, P. Vontobel, E. Lehmann, and R. Schulin. 2008. Quantitative imaging of infiltration, root growth, and root water uptake via neutron radiography. Vadose Zone J. 7:10351047. doi:10.2136/vzj2007.0156

Pohlmeier, A., M. Javaux, $H$. Vereecken, S. Haber-Pohlmeier, S.H. Anderson, and J.W. Hopmans. 2013. Magnetic resonance imaging techniques for visualization of root growth and root water uptake processes In: S.H. Anderson and J.W. Hopmans, editors, Soil-waterroot processes: Advances in tomography and imaging. SSSA Spec. Publ. 61. SSSA, Madison, WI. p. 137-156.

Read, D.B., A.G. Bengough, P.J. Gregory, J.W. Crawford, D. Robinson, C.M Scrimgeour, et al. 2003. Plant roots release phospholipid surfactants that modify the physical and chemical properties of soil. New Phytol. 157:315-326. doi:10.1046/j.1469-8137.2003.00665.x

Simovic, S. S. Tamburic, J. Milic-Askrabic, and D. Rajic. 1999. An investigation into interactions between polyacrylic polymers and a non-ionic surfactant: An emulsion preformulation study. Int. J. Pharm. 184:207-217. doi:10.1016/S0378-5173(99)00097-6

Sposito, G. 2013. Green water and global food security. Vadose Zone J. 12(4). doi:10.2136/vzj2013.02.0041

Tardieu, F., and B. Parent. 2017. Predictable "meta-mechanisms" emerge from feedbacks between transpiration and plant growth and cannot be simply deduced from short-term mechanisms. Plant Cell Environ. 40:846-857. doi:10.1111/pce.12822

Tron, S., G. Bodner, F. Laio, L. Ridolfi, and D. Leitner. 2015. Can diversity in root architecture explain plant water use efficiency? A modeling study. Ecol. Modell. 312:200-210. doi:10.1016/j.ecolmodel.2015.05.028
Tumlinson, L.G., H. Liu, W.K. Silk, and J.W. Hopmans. 2008. Thermal neutron computed tomography of soil water and plant roots. Soil Sci. Soc. Am J. 72:1234-1242. doi:10.2136/sssaj2007.0302

Wasson, A.P., R.A. Richards, R. Chatrath, S.C. Misra, S.V.S. Prasad, G.J. Rebetzke, et al. 2012. Traits and selection strategies to improve root systems and water uptake in water-limited wheat crops. J. Exp. Bot. 63:3485-3498. doi:10.1093/jxb/ers 111

Watt, M., M.E. McCully, and M.J. Canny. 1994. Formation and stabilization of rhizosheaths of Zea mays L. (Effect of soil water content). Plant Physiol. 106:179-186. doi:10.1104/pp.106.1.179

York, L.M., A. Carminati, S.J. Mooney, K. Ritz, and M.J. Bennett. 2016. The holistic rhizosphere: Integrating zones, processes, and semantics in the soil influenced by roots. J. Exp. Bot. 67:3629-3643. doi:10.1093/jxb/erw108

Young, I.M. 1995. Variation in moisture contents between bulk soil and the rhizosheath of wheat (Triticum aestivum L. Cv. Wembley). New Phytol. 130:135-139. doi:10.1111/j.1469-8137.1995.tb01823.x

Zarebanadkouki, M., M.A. Ahmed, and A. Carminati. 2016. Hydraulic conductivity of the root-soil interface of lupin in sandy soil after drying and rewetting. Plant Soil 398:267-280. doi:10.1007/s $11104-015-2668-1$

Zarebanadkouki, M., and A. Carminati. 2014. Reduced root water uptake after drying and rewetting. J. Plant Nutr. Soil Sci. 177:227-236. doi:10.1002/jpln.201300249

Zickenrott, I.-M., S.K. Woche, J. Bachmann, M.A. Ahmed, and D. Vetterlein. 2016. An efficient method for the collection of root mucilage from different plant species: A case study on the effect of mucilage on soil water repellency. J. Plant Nutr. Soil Sci. 179:294-302. doi:10.1002/jpln.201500511 